\title{
LOS ESTUDIANTES DE EDUCACIÓN SECUNDARIA Y EL MEDIO AMBIENTE
}

\author{
Raul Calixto Flores ${ }^{1}$
}

Resumen: En este artículo se describen los principales resultados de una investigación efectuada con estudiantes de cuatro escuelas secundarias (una general, una técnica y dos telesecundarias) del estado de Jalisco (México), con el objetivo de identificar las representaciones sociales (RS) del medio ambiente. La importancia de este estudio, radica en que nos permite comprender que las representaciones son producto de un proceso de socialización, en el que la escolarización contribuye a la selección, transformación y organización de los conocimientos cotidianos.

En esta investigación se sigue una propuesta metodológica que deriva de los trabajos de Moscovici y Jodelet (1986), recuperando el discurso escrito de los estudiantes por medio de la encuesta y las cartas asociativas. El cuestionario de la encuesta se fundamenta en los trabajos de Grize, Verges, y Silem (1987), construido para la evocación de las RS y la carta asociativa a partir del cuestionario de asociación perceptivo-conceptual de Peña (1997). En los resultados obtenidos se observa en los estudiantes un mayor número de representaciones naturalistas y antropocéntricas pactuadas del medio ambiente. Una de las conclusiones derivadas del estudio destaca la relación de las representaciones sociales con prácticas específicas de educación ambiental.

Palabras clave: Representaciones sociales, medio ambiente, investigación, educación ambiental

\section{ESTUDANTES DO ENSINO FUNDAMENTAL II E DO MEIO AMBIENTE}

Resumo: Este artigo descreve os principais resultados de uma pesquisa realizada com alunos de quatro escolas de ensino fundamental II (uma geral, uma técnica e duas tele-escolas ${ }^{2}$ ), no estado de Jalisco (México), a fim de identificar as representações sociais (RS) do meio ambiente. A importância deste estudo é que vamos entender que as representações são o produto de um processo de socialização em que a escolaridade contribui para a seleção, transformação, e organização do conhecimento cotidiano.

Esta pesquisa segue uma metodologia derivada do trabalho de Moscovici e Jodelet (1986), recuperando o discurso escrito dos alunos através de uma pesquisa e de cartas associativas. O questionário da pesquisa é baseado na obra de Grize, Verges, e Silem (1987), construído para a evocação das RS, e a carta associativa a partir do questionário de associação perceptivo-conceitual de Peña (1997). Os resultados obtidos mostram que os alunos tem um maior número de representações naturalistas e antropocêntricas

\footnotetext{
1 Universidad Pedagógica Nacional, Tlalpan, Mexico, calixtoflores@hotmail.com.

2 Modalidade educativa que estabelece seus centros escolares em zonas de alta marginalidade social, com um professor que atende todas as matérias e conta com recursos áudio-visuais, informáticos e impressos como apoio às diferentes matérias. Surge para levar a educação a lugares distantes e dispersos com inclusão e democracia.
} 
pactuadas do meio ambiente. Uma das conclusões do estudo destaca a relação das representações sociais com práticas específicas da educação ambiental.

Palavras-chave: Representações sociais, meio ambiente, pesquisa, educação ambiental

\title{
SECONDARY EDUCATION STUDENTS AND THE ENVIRONMENT
}

\begin{abstract}
This article describes the main results of an investigation conducted with students from four high schools (one general, one technical and two secondary schools) in the state of Jalisco (Mexico), with the aim to identify the social representations of the environment. The importance of this study is that let us to understand that representations are the product of a socialization process in which schooling contributes to the selection, transformation and organization of everyday knowledge.

This research follows a methodology derived from the work of Moscovici and Jodelet (1986), recovering the written speech of students through the survey and associative letters. The survey questionnaire is based on the work of Grize, Verges, and Silem (1987), built for the evocation of the RS and the letter from the questionnaire associative perceptual-conceptual association of Peña (1997). The results obtained show the students a greater number of naturalists and anthropocentric "pactuadas" representations of the environment. One conclusion from the study highlights the relationship of social representations specific environmental education practices.
\end{abstract}

Keywords: Social representations, environment, research, environmental education

\section{Introducción}

La investigación que se describe en este artículo se desarrolló en el estado de Jalisco, localizado en la zona occidente de la República Mexicana, que tiene una extensión de 80.137 $\mathrm{km} 2$, lo que representa el $4.09 \%$ del total de la superficie del territorio del país. Se encuentra limitado al noroeste con Nayarit; al noreste con Guanajuato y San Luis Potosí; al sur con Colima; al sureste con Michoacán y al suroeste con el Océano Pacífico.

En el Estado de Jalisco existen varios problemas ambientales, entre otros, el deficiente manejo de residuos sólidos, la deforestación acelerada y la pérdida creciente de agua potable. De acuerdo con la información de la Comisión Estatal de Ecología, ninguno de los 124 municipios del Estado, cuenta con lugares apropiados para dar un destino final a los residuos sólidos de origen doméstico, comercial o industrial no peligrosos.

Además, en las zonas forestales se observa la tala inmoderada, la quema de pastizales, el uso inadecuado de técnicas, así como los cambios en el uso del suelo. Existe una demanda creciente del uso de agua que no corresponde a su cuidado, ya que no se recicla, se desperdicia y se contamina.

Estos problemas ambientales están presentes en la vida cotidiana de los estudiantes de las escuelas secundarias de Jalisco y constituyen un referente importante para la conformación de sus RS del medio ambiente. Otro referente relevante son los contenidos presentes en los programas de estudio de la escuela secundaria y los proyectos referentes al medio ambiente que se desarrollan en ellas. En este marco, el objetivo fue conocer las representaciones sociales (RS) de los estudiantes de este nivel educativo respecto al medio ambiente.

El artículo comprende cuatro secciones: la primera describe los referentes teóricos de la educación ambiental y las representaciones sociales; la segunda da cuenta del proceder 
metodológico; la tercera analiza los principales resultados; y la cuarta presenta las conclusiones. Por último, algunas reflexiones finales complementan el artículo.

\section{Educación ambiental y representaciones sociales}

En México la instauración de la educación ambiental se remonta a 1982, con la creación de la Secretaría de Desarrollo Urbano y Ecología (SEDUE) y la promulgación de la Ley Federal de Protección al Ambiente. Desde su institucionalización en el sistema escolar, se han desarrollado numerosos planes, programas y proyectos, muchos de éstos generados por la iniciativa de las propias comunidades educativas, y otros más impulsados por instituciones como el Centro de Educación y Capacitación para el Desarrollo Sustentable (CECADESU).

La educación ambiental comprende una serie de temas y contenidos que son abordados desde los primeros niveles escolares (educación preescolar).

En la Reforma de la Educación Secundaria iniciada en 2006, la educación ambiental es considerada en forma transversal, proponiendo contenidos referidos al medio ambiente en distintas asignaturas.

Las asignaturas de Ciencias 1 (Biología), Ciencias 2 (Física) y Ciencias 3 (Química), Geografía, Formación Cívica, además de la materia estatal y Tutoría, principalmente, constituyen un referente importante para que los estudiantes de educación secundaria construyan sus representaciones sobre el medio ambiente. Además, a través de diversas fuentes de información como la televisión-, los estudiantes tienen acceso a temas relacionados con el medio ambiente, que contribuyen también a la formación de estas representaciones. En este marco se puede afirmar que los estudiantes de educación secundaria poseen representaciones que es necesario conocer y caracterizar como una forma de valorar los alcances de la educación ambiental.

La incorporación de la educación ambiental al sistema escolar resulta de la preocupación creciente por los efectos negativos de la destrucción ambiental en la calidad de vida de las personas. Esto ocurre a partir de la toma de conciencia de los efectos nocivos para la salud humana y para todas las formas de vida, ocasionados por los residuos tóxicos y pesticidas denunciados, entre otros por Rachel Carson (1962).

Esta toma de conciencia originó nuevas demandas para diversas instituciones, como las escolares, que gradualmente fueron incorporando contenidos, estrategias y asignaturas referidas no sólo a nuevos conocimientos, sino también al fortalecimiento de valores y actitudes para formar una ciudadanía ambientalmente responsable.

Para Enrique Leff, la educación ambiental conlleva la formación de nuevas actitudes, lo que implica:

[...] educar para formar un pensamiento crítico, creativo y prospectivo, capaz de analizar las complejas relaciones entre procesos naturales y sociales, para actuar en el ambiente con una perspectiva global, pero diferenciada por las diversas condiciones naturales y culturales que lo definen (Leff, 1998, p.217).

La educación ambiental se da en distintos ámbitos; sin embargo, en las instituciones escolares tiene lugar un proceso planificado y sistematizado de experiencias de aprendizajes, que conduce hacia el logro de los objetivos de este tipo de educación. En la actualidad, como lo refiere Marco Reigota (2007), la educación ambiental, cada vez más aborda temas conflictivos, que tocan intereses políticos de diversa naturaleza, como los relacionados con los transgénicos, la biodiversidad, la salud mental en las ciudades y los saberes tradicionales de los diversos grupos étnicos.

Una de las teorías que posibilita comprender el pensamiento social de los sujetos sobre diversos aspectos de la educación, es la teoría de las RS. Y, específicamente, sobre la educación ambiental, ya que es una de las teorías más pertinentes para comprender el sentido y significado 
que se le confiere al medio ambiente.

Las RS son un tema importante para la Educación Ambiental. En cierto sentido, la materia prima con la que trabajamos los educadores y las educadoras ambientales, aquello sobre lo que y con lo que construimos nuestras prácticas y establecemos objetivos más o menos ambiciosos de cambio, son las representaciones sociales que tienen los destinatarios de nuestras acciones -individuos o colectivos sociales- sobre determinados problemas ambientales o sobre la crisis ambiental en general. Es a partir de estas representaciones construidas individual y socialmente que podemos dotar de significado y de sentido al mundo que nos rodea, compartirlo con otros y orientar nuestras actitudes $y$ comportamientos con respecto a él (Meira, 2002, p. 94).

Las RS corresponden a una forma de ver el mundo, con una serie de nociones que permiten identificar la visión que tiene el sujeto acerca de cierto objeto. Por una parte, esta teoría critica las posturas clásicas de la psicología conductual, donde la relación sujeto-objeto está basada solo en la relación estímulo-respuesta; pero, por otra parte, afirma que en la relación entre el objeto y el sujeto existe una relación interdependiente.

Serge Moscovici, creador de la teoría de las RS, las define como:

Sistemas de valores, ideas y prácticas que tienen una doble función: en primer lugar, establecer un orden que permita a los individuos orientarse en un mundo social y material, y dominarlo; y, en segundo término, permitir la comunicación entre los miembros de una comunidad, aportándoles un código para el intercambio social y un código para denominar y clasificar de manera inequívoca los distintos aspectos de su mundo y de su historia individual y grupal (Moscovici, 1973, p. XIII).

Las RS se orientan hacia la comunicación, la comprensión y el dominio del entorno material y social. Son construcciones de los sujetos sobre un objeto, pero nunca reproducciones de ese objeto.

La representación es una noción creada para explicar qué es lo que une a la gente en un grupo, en una sociedad y los hace actuar. Para estar juntos, crear instituciones y aceptar reglas comunes, la gente debe compartir un sistema de creencias y representaciones comunes distintivas (típicas) de su propia cultura (Augusto Palmonari, 2004).

Las RS del medio ambiente pueden entenderse como las formulaciones sintéticas de sentido descriptible y diferenciable (Mato, 1999), es decir, las RS del medio ambiente son construcciones sociales, que resultan de las ideas que circulan, se transforman y dan sentido a las relaciones intersubjetivas.

Entre otras investigaciones que tienen como referente teórico las RS, y que fueron hechas en el campo de la educación ambiental y en el nivel de educación básica, se encuentran las desarrolladas por Esperanza Terrón (2010), Beatriz Gutiérrez (2011) y Rosa Elvira Valdez (2011).

Las RS que se encuentran en la educación ambiental, en el nivel de educación básica, tienen que ver con la manera en que los sujetos -estudiantes y profesores- aprenden los acontecimientos de la vida diaria, las informaciones que poseen (principalmente de los planes y programas de estudios) y las características del medio ambiente que observan.

\section{Proceder metodológico}

El estado de Jalisco tiene un total de 1911 escuelas secundarias, de éstas 764 son secundarias generales, 317 son secundarias técnicas y 638 son telesecundarias.

La investigación se llevó a cabo en: la Escuela Secundaria General Vicente Guerrero, en la Escuela Secundaria Técnica 145, en la Telesecundaria Adolfo López Mateos y en la 
Telesecundaria Julián Carrillo. En la investigación que se describe en el presente trabajo, predomina el enfoque interpretativo, con utilización de dos técnicas: encuesta y carta asociativa. El cuestionario utilizado se fundamenta en la propuesta de Grize, Verges, y Silem (1987), y la carta asociativa a partir del cuestionario de asociación perceptivo conceptual de Peña (1997).

Con las respuestas de los cuestionarios fue posible analizar el lenguaje empleado en el sentido común, identificando la dispersión de la información, centrándonos en la focalización y la presión de la inferencia. La focalización permite determinar los intereses centrales y la presión de la inferencia, con la cual se reconocen las posturas y acciones derivadas de los intereses centrales.

Con las cartas asociativas se encontró la dimensión de información de las RS, llegando a generar representaciones gráficas del medio ambiente.

En dicha investigación prevalece el supuesto de que la realidad se construye socialmente a través de definiciones de la situación, de naturaleza individual o colectiva. En este estudio nos interesó descubrir los significados no manifiestos de la experiencia vivida por los estudiantes, así como la estructura de las representaciones. Para ello se empleó la propuesta metodológica de Clémence (2001), que comprende tres fases: en la primera se conforma el mapa de los puntos de referencia compartidos y se identifican los contenidos que circulan con relación al medio ambiente. La segunda fase se centra en reconocer los principios que organizan las variadas posiciones individuales o los grupos que se investigan. Los datos recolectados permiten realizar comparaciones intergrupales (entre las escuelas secundarias, en este caso).

En la tercera fase se propone la formación de grupos a partir de la información producida mediante los cuestionarios. En esta fase se analizan los vínculos entre posiciones y principios con las características de los informantes.

La investigación se apoyó en materiales lingüísticos, sobre todo, en conjuntos de palabras jerarquizadas y producidas en cuestionarios o asociaciones de palabras, y los datos se analizaron para descubrir variaciones intergrupales. El lenguaje es la vía natural para identificar las RS de los estudiantes; facilita la comunicación intersubjetiva, fomenta los procesos intrasubjetivos y contribuye a ordenar la realidad. Las representaciones son expresadas por el lenguaje y es la expresión de estas la que produce trayectos más largos y más complejos para las correspondencias entre categorías del entorno, cada vez más ricas, y categorías de acción de los sujetos, cada vez más complicadas (Piñuel y Gaitán, 1999).

El análisis de los cuestionarios implicó varios procesos, de los que se obtienen la frecuencia de los términos utilizados, para después conocer su rango de aparición en la asociación $y$, finalmente, la importancia que tienen para los estudiantes. Las cartas asociativas se analizan con referencia a la relación asociativa y no respecto a los términos. Primero sobre la serie de asociaciones, después sobre el conjunto (de asociaciones), para concluir con el conjunto de asociaciones producidas.

\section{Resultados}

La investigación se realizó en cuatro distintas regiones del Estado, dos de ellas en un medio urbano y las otras dos de un medio rural.

Los principales resultados se sintetizan en la siguiente tabla:

\begin{tabular}{|l|l|l|l|l|}
\hline $\begin{array}{l}\text { Institución / nú- } \\
\text { mero de estudian- } \\
\text { tes participantes } \\
\text { en el estudio (n) }\end{array}$ & $\begin{array}{l}\text { Representacio- } \\
\text { nes predomi- } \\
\text { nantes }\end{array}$ & $\begin{array}{l}\text { Principales } \\
\text { elementos }\end{array}$ & $\begin{array}{l}\text { Problemas } \\
\text { ambientales }\end{array}$ & $\begin{array}{l}\text { Fuentes de } \\
\text { información }\end{array}$ \\
\hline $\begin{array}{l}\text { Escuela secundaria } \\
\text { Vicente Guerrero } \\
(\mathrm{n}=73)\end{array}$ & $\begin{array}{l}\text { Naturalistas } \\
\text { Antropocén- } \\
\text { tricas pactuadas }\end{array}$ & $\begin{array}{l}\text { Naturaleza, seres } \\
\text { vivos, ambiente, } \\
\text { biodiversidad, } \\
\text { contaminación }\end{array}$ & $\begin{array}{l}\text { Contaminación del } \\
\text { agua; generación de } \\
\text { basura }\end{array}$ & $\begin{array}{l}\text { Televisión, } \\
\text { escuela, } \\
\text { asignaturas }\end{array}$ \\
\hline
\end{tabular}




\begin{tabular}{|l|l|l|l|l|}
\hline $\begin{array}{l}\text { Escuela secundaria } \\
\text { técnica 145 } \\
(\mathrm{n}=131)\end{array}$ & $\begin{array}{l}\text { Naturalistas } \\
\text { Antropocén- } \\
\text { tricas pactuadas }\end{array}$ & $\begin{array}{l}\text { Entorno, } \\
\text { naturaleza, flora }\end{array}$ & $\begin{array}{l}\text { Contaminación, } \\
\text { generación de } \\
\text { basura, } \\
\text { deforestación }\end{array}$ & $\begin{array}{l}\text { Televisión, } \\
\text { escuela, } \\
\text { asignaturas }\end{array}$ \\
\hline $\begin{array}{l}\text { Telesecundaria } \\
\text { Adolfo López } \\
\text { Mateos } \\
(\mathrm{n}=68)\end{array}$ & Naturalistas & $\begin{array}{l}\text { Naturaleza, agua, } \\
\text { fauna }\end{array}$ & $\begin{array}{l}\text { Contaminación, } \\
\text { generación de } \\
\text { basura, } \\
\text { deforestación }\end{array}$ & $\begin{array}{l}\text { Escuela, } \\
\text { televisión, familia }\end{array}$ \\
\hline $\begin{array}{l}\text { Telesecundaria } \\
\text { Julián Carrillo } \\
(\mathrm{n}=133)\end{array}$ & $\begin{array}{l}\text { Naturalistas } \\
\text { Antropocén- } \\
\text { tricas pactuadas }\end{array}$ & Agua, flora, fauna & $\begin{array}{l}\text { Contaminación, } \\
\text { generación de } \\
\text { basura, } \\
\text { deforestación }\end{array}$ & $\begin{array}{l}\text { Escuela, } \\
\text { televisión, familia }\end{array}$ \\
\hline
\end{tabular}

Tabla. Resumen de los principales resultados. Las escuelas elegidas para el estudio corresponden a distitintas modalidades de enseñanza: diurna, técnica y telesecundaria; y a dos contextos diferentes: urbano (Escuela Secundaria Diurna Vicente Guerrero, Secundaria Técnica 145) y rural (Telesecundarias Adolfo López Mateos y Julián Carrillo).

\section{Las RS de los estudiantes de la Escuela Secundaria Diurna Vicente Guerrero}

La Escuela Secundaria Vicente Guerrero se localiza en La Huerta, Jalisco, en la región suroeste del estado y forma parte de la zona costera del mismo, colindando con los Municipios de Villa de Purificación, Casimiro Castillo, Cuautitlán de García Barragán, Tomatlán y Cihuatlán.

El total de alumnos que participó en la investigación fue de 73 , de los cuales 33 son mujeres y 40 hombres, con un promedio de edad de 13.98 años; 20 estudian el primer grado y 53 el segundo grado. Respecto a su conocimiento sobre qué es el medio ambiente, 66 estudiantes contestaron afirmativamente y 7 de forma negativa; esta proporción cambió cuando se les preguntó sobre la sustentabilidad: 44 estudiantes contestaron de manera positiva y 29 de forma negativa.

$\mathrm{Al}$ analizar las representaciones sobre el medio ambiente, se observa el predominio de las RS naturalistas, ya que 50 estudiantes utilizaron términos de las ciencias naturales para explicar el medio ambiente y un número menor (38) alude a algún problema ambiental. El término que se utilizó con mayor frecuencia fue "naturaleza" (19 estudiantes). Pero al preguntar sobre sustentabilidad, no se observó claridad en las respuestas y el término que utilizaron con mayor frecuencia (9 estudiantes) fue el de "sostener".

$\mathrm{Al}$ profundizar respecto al medio ambiente, los alumnos emplean en su lenguaje términos como "basura" (12 estudiantes) o "contaminación del agua" (12). Términos que corresponden a las representaciones sociales pactuadas del medio ambiente.

Por otra parte, los estudiantes realizan una apreciación de las condiciones en las que se encuentra el medio ambiente en la familia, la escuela, la comunidad y el planeta.

Los estudiantes señalan la existencia de muchos problemas ambientales en la región, como la contaminación (30 estudiantes), generación de basura (13) y la tala de árboles (10). Al preguntarles sobre el aspecto que más les disgusta del medio ambiente, la mayoría de los estudiantes se refiere a la contaminación (31) y lo que más les gusta es la existencia de animales en la región (33).

Las actitudes de los estudiantes son favorables cuando mencionan el medio ambiente en la familia y escuela, pero no es así cuando se vinculan a la comunidad y al planeta. Al interrogarlos sobre las condiciones en que se encuentra el medio ambiente en el planeta, se verifica que para 18 estudiantes estas son mejores, para 8 son iguales y para 47 son peores.

Los alumnos observan varias acciones favorables para cuidar el medio ambiente, las que presentan mayor frecuencia son las de "no tirar basura" (31 estudiantes) y "no desperdiciar el 
agua" (16).

Y las acciones no favorables con mayor frecuencia son la de "tirar basura" (42 estudiantes) y "maltratar a los animales" (13). En estas respuestas se identifica el predominio de las RS naturalistas y RS antropocéntricas pactuadas, al referirse sobre todo a elementos de la naturaleza y a las cuestiones que impactan de forma no favorable al ser humano.

Las RS que los estudiantes poseen sobre el medio ambiente se configuran de acuerdo con el discurso empleado por ellos.

RS naturalistas. Elementos centrales: seres vivos, ambiente y biodiversidad; elementos secundarios: naturaleza, planeta y ecosistema; elementos terciarios: aire, bosques, suelo y agua.

RS antropocéntricas pactuadas. Elementos centrales: contaminación; elementos secundarios: basura, agua, tala de árboles y maltrato de animales; elementos terciarios: explotación, agotamiento y hambre.

Las RS tienen su origen en diversas fuentes, las que se señalan con mayor frecuencia son la televisión (55 estudiantes), la escuela (51), las asignaturas que trabajan en las clases (46) y en conversación con su familia (30).

Existe información sobre el medio ambiente y sus problemas, aunque los estudiantes cuentan con poca información respecto a la sustentabilidad.

Las asignaturas referidas por los estudiantes, donde se abordan estos temas son: Ciencias 1, Biología (62 estudiantes), Geografía (44), y Formación Cívica (23).

\section{Las RS de los estudiantes de la Escuela Secundaria Técnica 145}

Esta secundaria se localiza en la colonia el Sauz, que se encuentra entre los municipios de Guadalajara y Tlaquepaque, y se divide en cuatro barrios, que a pesar de contar con todos los servicios básicos, es una zona de gran hacinamiento humano.

El total de participantes en la Escuela Secundaria Técnica 145 fue de 131, de los cuales 60 son mujeres y 71 son hombres, con un promedio de edad de 13.32 años y todos estudian el segundo grado. Respecto a su conocimiento sobre qué es el medio ambiente, 105 estudiantes contestaron afirmativamente y 26 de forma negativa.

$\mathrm{Al}$ analizar las representaciones sobre el medio ambiente, se observa el predominio de las RS naturalistas. Los estudiantes utilizan términos relacionados con las ciencias naturales, siendo los más frecuentes "entorno" (42 estudiantes), "naturaleza" (39) y "flora" (21).

Sin embargo, al igual que los estudiantes de la Secundaria Vicente Guerrero, al abordar la sustentabilidad hay muchas confusiones y la gran mayoría no contesta (98 estudiantes). Y las respuestas de los 33 restantes son muy diversas, destacando el empleo de los términos "mantener" (17 estudiantes) y "dinero" (15).

En cuanto a las RS del medio ambiente, resalta la información prevaleciente en los estudiantes. Esa información está relacionada con conceptos biológicos, como: flora (21 estudiantes), agua (17), fauna (16), aire (15) y oxígeno (15).

Los alumnos señalan entre los problemas ambientales la contaminación (62 estudiantes), el de generación de basura (32) y también el problema de la deforestación (19).

Los estudiantes tienen actitudes favorables cuando se relacionan al medio ambiente en la familia y la escuela, pero no es así cuando se vinculan con la comunidad y el planeta. Al preguntar sobre las condiciones en las que se encuentra el medio ambiente en el planeta, se verifica que para 25 estudiantes estas son mejores, para 30 estudiantes son iguales y para 72 son peores.

Estas respuestas son preocupantes, ya que en las representaciones de los estudiantes prevalecen los aspectos no favorables del medio ambiente.

Los estudiantes encuentran varias acciones favorables para cuidar el medio ambiente, teniendo mayor frecuencia las de no tirar basura (66 estudiantes) y las de separar la basura (28).

En cuanto a las acciones no favorables, tienen mayor frecuencia tirar basura (78 estudiantes), maltratar la flora (32) y desperdiciar el agua (18). 
Se observan elementos de las RS naturalistas y RS antropocéntricas pactuadas en los estudiantes, al referirse sobre todo a elementos de la naturaleza y a las relacionadas con cuestiones que impactan de forma no favorable al ser humano.

De acuerdo con el discurso empleado por los estudiantes, se configuran las RS que estos poseen sobre el medio ambiente.

RS naturalistas. Elementos centrales: biodiversidad, seres vivos y naturaleza; elementos secundarios: ecosistemas, flora, fauna y oxígeno; elementos terciarios: bosques, aire, agua y suelo.

RS antropocéntricas pactuadas. Elementos centrales: pobreza, ciencia y contaminación; elementos secundarios: planeta, hambre y explotación; elementos terciarios: aerosoles, tala de árboles y cacería de animales.

Las RS tienen su origen en diversas fuentes; las que se identifican con mayor frecuencia son la televisión (116 estudiantes), la escuela (77), las asignaturas que trabajan en las clases (62) y en conversación con su familia (51).

Aunque existe información sobre el medio ambiente y sus problemas, los estudiantes tienen poca información a respecto de la sustentabilidad.

Las asignaturas que abordan estos temas a los que se refieren los estudiantes son Ciencias 1 , Biología (106 estudiantes), Español (49), Formación Cívica (37), Geografía (36) y Orientación (36).

En los estudiantes de educación secundaria se observan elementos de las RS naturalistas en el campo de representación del medio ambiente y RS antropocéntricas pactuadas en el campo de representación de la sustentabilidad.

\section{Las RS de los estudiantes de la Telesecundaria Adolfo López Mateos}

Esta telesecundaria se encuentra en la comunidad de Agua Caliente, Municipio de Poncitlán, Jalisco, en la ribera del lago de Chapala, región ciénega del estado de Jalisco y cuenta con una población de 1100 habitantes. El acceso a esta comunidad es complicado debido a lo escarpado del terreno y lo sinuoso de la brecha, misma que en tiempos de lluvia queda totalmente destruida, lo que obliga a pobladores y personas que laboran en la comunidad a entrar caminando o en lancha.

El total de estudiantes de la Telesecundaria Adolfo López Mateos que participó fue de 68, de los cuales 23 son mujeres y 45 hombres, con un promedio de edad de 13.5 años; 32 estudiantes cursan el primer grado, 18 el segundo y 18 el tercero. Respecto a su conocimiento sobre qué es el medio ambiente, 64 estudiantes contestaron afirmativamente y sólo 4 dijeron que no.

En cuanto a los términos que constituyen las RS del medio ambiente, se observa que prevalecen los relacionados con las ciencias naturales. Predominan los siguientes términos: "naturaleza" (28 estudiantes), "agua" (13) y "fauna" (11). Respecto a la existencia de representaciones sobre la sustentabilidad, no logran identificarse elementos que las constituyan. La sustentabilidad constituye un aspecto desconocido, algunos estudiantes la vinculan con la elaboración de un proyecto (4 estudiantes) y el manejo de recursos (4).

Respecto a las RS del medio ambiente, la información prevaleciente en los estudiantes está relacionada a conceptos biológicos como "naturaleza" (18 estudiantes) "conservación" (16) y "vida" (14). Pero también a conceptos asociados con el impacto del medio ambiente en la vida del ser humano: "contaminación" (14) y "quema de la vegetación en terrenos" (6).

Los estudiantes identifican entre los principales problemas ambientales, el de la contaminación (62 estudiantes), el de la generación de basura (32), así como el problema de la deforestación (19).

$\mathrm{Al}$ referirse a las acciones favorables ( $\mathrm{f}$ ) y no favorables (nf) que se hacen en torno al medio ambiente, tienen mayor apreciación las acciones favorables. 
Las acciones favorables se observan más en la escuela y la familia, y menos en la comunidad y el planeta, donde sobresalen las acciones no favorables. Respecto a las condiciones en que se encuentra el medio ambiente en el planeta, para 21 estudiantes son mejores, para 18 son iguales y para 29 son peores.

El conjunto de respuestas anteriormente señaladas revelan que en las representaciones de los estudiantes prevalecen los aspectos no favorables del medio ambiente.

Los estudiantes reconocen la práctica de acciones favorables, como 'no tirar basura' (33 estudiantes), 'cuidar las plantas' (14) y 'evitar la quema de basura' (9). En tanto que en las acciones no favorables aparecen con mayor frecuencia 'tirar basura' (19 estudiantes), 'quemar basura' (13), 'desperdiciar agua' (12) y 'la deforestación' (10).

Tomando en cuenta sus respuestas, se configura la presencia de un tipo de representaciones naturalistas en los estudiantes, ya que se hace evidente el uso de términos asociados a la naturaleza.

RS naturalistas. Elementos centrales: naturaleza, agua y vida; elementos secundarios: cuidar y aire; elementos terciarios: seres vivos, biodiversidad y ecosistema.

Las RS tienen su origen en diversas fuentes, para la mayoría de los estudiantes de esta telesecundaria, la principal fuente de información es su propia escuela (43 estudiantes), la televisión (30), la familia (19) y los libros (19).

Existe información sobre el medio ambiente y sus problemas; sin embargo, los estudiantes tienen poca información respecto a la sustentabilidad.

Las asignaturas que refieren los estudiantes, donde se abordan estos temas son Ciencias 1, Biología (106 estudiantes), Español (49), Formación Cívica (37), Geografía (36) y Orientación (36).

\section{RS de los estudiantes de la Telesecundaria Julián Carrillo}

Esta escuela telesecundaria se localiza en la población de Telcruz, municipio de Cuautitlán de García Barragán en el Estado de Jalisco; en la región de la Costa Sur, cerca de la cadena montañosa de la Sierra Madre de Occidente en México.

El total de participantes de la telesecundaria Julián Carrillo fue de 133, de los cuales 64 son mujeres y 69 son hombres, con un promedio de edad de 13.77 años; 46 estudiantes son de primer grado, 40 de segundo y 47 de tercero. Respecto a su conocimiento sobre qué es el medio ambiente, 128 estudiantes contestaron afirmativamente y 5 de forma negativa.

En los estudiantes predominan las RS naturalistas, ya que al referirse al medio ambiente prevalecen los siguientes términos: agua (45 estudiantes), flora y fauna (32), naturaleza (16) y Tierra (13).

Con relación a las apreciaciones que los estudiantes hacen de las condiciones en las que se encuentra el medio ambiente, se observa que con mayor frecuencia califican como deficiente la condición en la comunidad y el planeta.

Estas respuestas corresponden a los problemas ambientales que señalan los estudiantes; la mayoría de ellos identifica el problema de la contaminación (62 estudiantes), también el de generación de basura (32) y el problema de la deforestación (19).

$\mathrm{Al}$ referirse a las acciones favorables ( $\mathrm{f}$ ) y no favorables ( $\mathrm{nf}$ ) que se realizan en torno al medio ambiente, los estudiantes observan un mayor número de acciones no favorables en la comunidad.

En el conjunto de respuestas de los estudiantes se observan actitudes no favorables hacia el medio ambiente. Se advierten apreciaciones diferenciadas, pero la tendencia predominante es un mayor reconocimiento de las acciones no favorables.

Los estudiantes encuentran varias acciones no favorables para cuidar el medio ambiente, las que tienen mayor frecuencia son: tirar basura (94 estudiantes), cortar árboles (39), desperdiciar el agua (36) y la contaminación del aire (31). 
En tanto que las acciones favorables con mayor frecuencia son las de sembrar árboles (38 estudiantes), cuidar el agua (36) y cuidar la naturaleza (32).

Se reconocen elementos de las RS naturalistas y RS antropocéntricas pactuadas en los estudiantes, al referirse sobre todo a elementos de la naturaleza y a las relacionadas con asuntos que impactan de forma no favorable al ser humano.

A partir de estos elementos es posible configurar las RS que poseen los estudiantes sobre el medio ambiente.

RS naturalistas. Elemento central: agua; elementos secundarios: entorno, árboles y aire; elementos terciarios: naturaleza, flora, fauna y suelo.

RS antropocéntricas pactuadas. El elemento central es la contaminación; elementos secundarios: planeta y deforestación; elementos terciarios: basura, pobreza y población.

Las RS tienen su origen en diversas fuentes, entre las que se encuentran con mayor frecuencia la escuela (129 estudiantes), la televisión (56), la familia (49) y los libros (42).

En cuanto a las asignaturas que los estudiantes señalan como portadoras de información respecto al medio ambiente son: Ciencias 1, Biología (113 estudiantes), Geografía (80), Tecnología (61), Estatal (56) y Español (48).

\section{Conclusiones}

En las investigaciones hechas sobre las RS en el campo de la educación ambiental, se observa la influencia teórica de Marcos Reigota, quien propone una tipología de RS que posteriormente ha sido retomada en la mayoría de las investigaciones. Reigota clasifica las RS como naturalistas, globalizantes y antropocéntricas. La RS naturalista se dirige a los aspectos físico-químicos y a la flora y fauna; la globalizante, cuando se consideran las interacciones entre los aspectos sociales y naturales; y la antropocéntrica, al orientarse hacia la utilidad de los recursos naturales para la vida del ser humano.

Hay que considerar el señalamiento de Tomás Ibáñez, en el sentido de que las RS “...no sólo inciden en la visión de la realidad social, sino también en su construcción efectiva..." (1994, p. 190). Si bien entre los estudiantes predominan las RS naturalistas, los comportamientos proambientales se orientan fundamentalmente a los aspectos conservacionistas del medio ambiente; estas representaciones dejan fuera el cuestionamiento de las formas en que se legitima la apropiación de los bienes naturales y comunes para toda la humanidad. En las RS antropocéntricas pactuadas prevalece un "puente" entre la sociedad y la naturaleza, e implican el reconocimiento de que las actividades humanas han impactado en el medio ambiente y se reconocen los efectos desfavorables de las actividades económicas y productivas de las sociedades modernas.

En las RS se discriminan elementos y problemas del medio ambiente, estableciendo correspondencias con las prácticas educativas. Las RS, a diferencia de otras formas de conocimiento de sentido común, pueden constituir una guía para el desarrollo de actividades prácticas de la educación ambiental. En cierto sentido, pueden ser la base para el desarrollo de determinados comportamientos proambientales.

Las RS naturalistas son las que se encontraron con mayor frecuencia en la investigación descrita; las que se vinculan con algunos enfoques de la educación ambiental resultaron ser las más comunes, tales como la educación para la conservación y la educación para la biodiversidad. Estas representaciones también predominan en los profesores de educación primaria (Terrón y González, 2009). En tanto Pedro Pablo Saleme, Alice Pereira de Faria y Jorge Luiz de Góes Pereira (2011) identifican en profesores y estudiantes de educación primaria el predominio de representaciones antropocéntricas. Aunque en los estudiantes universitarios (María Isabel Correa López y Benjamín Ortiz Espejel, 2012) predominan las representaciones antropocéntricas pactuadas.

El predominio de RS naturalistas y antropocéntricas pactuadas en los estudiantes de 
secundaria, permite reconocer las RS como aproximaciones de tomas de posición, en este caso, sobre el medio ambiente. La clasificación naturalista que predomina en los estudiantes demanda revisar los contenidos que las distintas asignaturas de la educación secundaria abordan con relación a la educación ambiental. Los contenidos pueden incorporar aspectos sobre el origen y consecuencias de los diversos problemas ambientales, así como también un análisis crítico, de las responsabilidades diferenciadas de los distintos sectores sociales, que propician la continuidad y, en ocasiones, la proliferación de estos problemas.

\section{Reflexiones finales}

En México la práctica y la investigación de la educación ambiental escolar manifiestan grandes dificultades y los resultados aún son incipientes. Es necesario, por tanto, abundar en la teoría e investigación en educación ambiental, recuperar y sistematizar experiencias para propiciar la integración de la educación ambiental en el currículo. La educación ambiental corresponde a un proceso educativo y cultural integrado a la formación del ser humano, que comienza en la infancia y continúa durante toda la vida. En las instituciones escolares se inicia una educación sistemática orientada al conocimiento de la naturaleza, la ecología, la complejidad de la relación de la sociedad con la naturaleza, los efectos de las actividades humanas sobre el medio ambiente y las acciones para cuidar nuestro entorno.

Las RS del medio ambiente son relevantes para comprender el sentido de la educación ambiental. En el caso de los estudiantes de educación secundaria, constituyen la base para asumir una posición frente a los problemas ambientales.

La teoría de las RS es importante para la educación ambiental, ya que contribuye a caracterizar la posición de los individuos que constituyen cada grupo, respecto a diversos aspectos, como la sustentabilidad y el medio ambiente. Existen diferentes enfoques para investigar las RS, el enfoque de aproximaciones de toma de posición, propuesto por Doise (1991) es relevante para la educación ambiental, ya que a través de las representaciones puede vincularse con la práctica de este campo, a partir de referencias comunes.

El papel que ocupan las RS del medio ambiente en el contexto de la educación ambiental permite hacer una valoración de los alcances de esta en las escuelas secundarias.

Las representaciones encontradas en este estudio son complementarias, ya que comprenden elementos articuladores.

Las representaciones son producto de un largo recorrido de acción y reflexión sobre el mundo, formando categorías o clases de entidades que tienen propiedades comunes, con lo cual se van construyendo los conceptos. Estos no son aislados, se forman redes de conceptos ligados a otros y hay una jerarquía entre ellos.

Marcos Reigota advierte que las RS pueden contener conceptos ingenuos o científicamente incorrectos, que al difundirse se consolidan o legitiman en la sociedad. En la presente investigación se verificó que los estudiantes consideran el medio ambiente como naturaleza. Representación fuertemente arraigada, que deja fuera los aspectos sociales y culturales del medio ambiente.

Según el enfoque de aproximaciones de toma de posición de la teoría de las RS, los estudiantes asumen una postura ante el medio ambiente, pero con relación a la educación ambiental; postura que puede traducirse después en comportamientos proambientales.

Así, de acuerdo con esta teoría, los estudiantes le atribuyen significados al medio ambiente, y por ende, esta será la orientación que le imprimirán en la práctica.

A partir del conocimiento de estas representaciones es posible proponer programas educativos que las transformen, ya que dejan fuera elementos relevantes para la educación ambiental. En la investigación descrita, no se identificaron RS globalizantes, en que se proponen la naturaleza y la 
sociedad como procesos continuos y mutuamente interdependientes, que nos conducen hacia la construcción de nuevos niveles de complejidad y diversidad. Uno de los retos de la educación ambiental, es el de transformar las RS naturalistas y antropocéntricas pactuadas, en RS globalizantes en los estudiantes, para que sean capaces de reconocer los procesos interdependientes entre la naturaleza y la sociedad.

\section{Referencias}

CORREA, Ma. Isabel y Benjamín Espejel. "Transformación de las RS a través de la educación ambiental en el nivel universitario", en La búsqueda de sentidos y significados de la educación ambiental (Raúl Calixto, coordinador), México, UPN, 2012.

DOISE, Willem. "Las representaciones sociales: presentación de un campo de investigación". Barcelona, Anthropos, 27, 1991.

DOISE, W., Alain Clémence y Fabio Lorenzi Cioldi. Representaciones sociales y análisis de datos. México, Instituto Mora, 1992.

GRIZE, Jean Blaise, Pierre Verges, Pierre, Ahmed Silem. Salaires face aux nouvelles technologies. Vers une approche sociologique des représentations sociales, Centre National de la Recherche Scientifique, France, 1987.

GONZÁLEZ, Gaudiano E. "La educación ambiental en México: logros, perspectivas y retos de cara al nuevo milenio", en memoria de Educación Ambiental para un Desarrollo Sustentable, Taller de Especialistas y 2o. Foro Nacional, México: SEMARNAT, ANEA, CESU, Universidad Autónoma de Aguascalientes, 2002.

GUTIÉRREZ, Beatriz. "Educación ambiental y pintura mural: una aproximación a las representaciones sociales del medio ambiente de los alumnos de educación primaria". En Memoria electrónica del XI Congreso Nacional de Investigación Educativa, México, Comie, 2011.

JODELET, Denise. "La representación social: fenómenos, conceptos y teoría" en Psicología Social II, Serge Moscovici, tr. David Rosenbaum, (edición original, 1984), Barcelona, pp. 469-534, 1986. LEEF, Enrique. Saber ambiental, México, Siglo XXI, 1998.

IBÁÑEZ, Tomás. Psicología social construccionista. México, Universidad de Guadalajara, 1994.

MEIRA, Pablo Ángel. "Problemas ambientales globales y educación ambiental: una aproximación desde las representaciones sociales del cambio climático”. En M. Campillo (ed.). Elpapel de la educación ambiental en la pedagogía social, Murcia, DM, p. 94, 2002.

MOSCOVICI, Serge. "Prólogo" a C. Herzlich, Health and Illness, Londres: Academia Press, 1973.

PALMONARI, Augusto. "Una mirada a la psicología social desde la perspectiva de la teoría de las representaciones sociales". En Eulogio Romero (ed.), Representaciones sociales. México, Benemérita Universidad Autónoma de Puebla, 2004. 
PEÑA. Antonio. La representación social del poder por estudiantes de psicología, Tesis de Maestría en Psicología Social, México, UNAM, 1997.

PIÑUEL, José Luis y Juan Antonio Gaitán. Metodología general. Conocimiento cientifico e investigación en la comunicación social, editorial Síntesis, Madrid, 1999.

REIGOTA, Marcos. "Les représentations sociales de l'environment et les pratiques pédagogiques quotiedennes des professeurs de Sciences a Sao Paulo-Brésill". Tesis de doctorado en Pedagogía de Biología. Louvain-la Neuve: Université Catholique de Louvain, 1990.

REIGOTA, Marcos. "As representacões sociais na prática pedagógica cotidiana da educação ambiental”. En L. Sauvé et al. (org). Textos escogidos en educación ambiental: de una América a la otra. Tome I. Montreal: Ere-Uqam, pp. 123-128, 2002.

REIGOTA, Marcos. "O estado da arte da pesquisa em educação ambiental no Brasil”, Pesquisa em educação ambiental, Vol. 2, No. 1, 33-66, 2007.

SALEME, Pedro Paulo; Pereira de Faria, Alice y de Góes Pereira, Jorge Luiz. Representações sociais de Meio Ambiente e educação ambiental nas escolas públicas do meio rural e urbano de Teófilo Otoni/MG. Revista Brasileira de Educaşão Ambiental (REVBEA), 6,(1), 35-40, 2011.

SAUVÉ, Lucie. "Uma cartografia das correntes em educação ambiental”. Em M. Sato, Carvalho, Isabel (ed.), A pesquisa em educação ambiental: cartografias de uma identidade narrativa em formação, Porto Alegre: Université du Québec à Montréal. Cátedra de investigación de Canadá en educación ambiental, 2004.

TERRÓN, Esperanza y González, Edgar. Representación y medio ambiente en la educación básica en México, Pesquisa em educação ambiental, Vol. 4. No. 1, 11-36, 2009.

TERRÓN, Esperanza. Educación ambiental. Representaciones sociales y sus implicaciones educativas, México, UPN, 2010.

VALDEZ, Rosa Elvira. "La educación ambiental en la escuela secundaria pública: su incorporación en Saltillo, Coahuila". En Memoria electrónica del XI Congreso Nacional de Investigación Educativa. México, Comie, 2011.

Artigo submetido em 31/01/2013

Artigo aprovado em 26/11/2013 